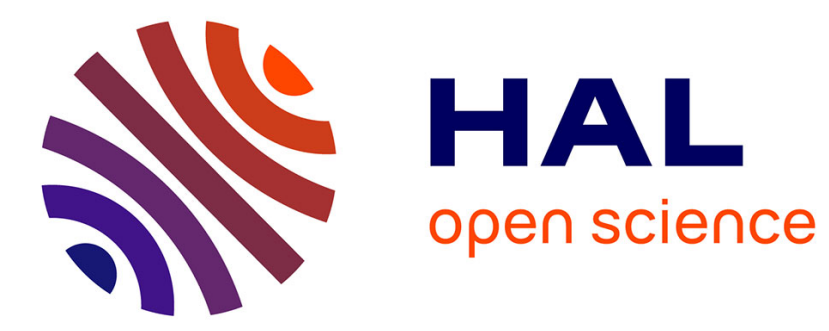

\title{
Robust Trajectory Planning of Under-Actuated Cable-Driven Parallel Robot with 3 Cables
}

\author{
Edoardo Idá, Sébastien Briot, Marco Carricato
}

\section{To cite this version:}

Edoardo Idá, Sébastien Briot, Marco Carricato. Robust Trajectory Planning of Under-Actuated CableDriven Parallel Robot with 3 Cables. Advances in Robot Kinematics (ARK 2020), Jun 2020, Ljubljana, Slovenia. hal-02539452

\section{HAL Id: hal-02539452 https://hal.science/hal-02539452}

Submitted on 22 Jun 2020

HAL is a multi-disciplinary open access archive for the deposit and dissemination of scientific research documents, whether they are published or not. The documents may come from teaching and research institutions in France or abroad, or from public or private research centers.
L'archive ouverte pluridisciplinaire HAL, est destinée au dépôt et à la diffusion de documents scientifiques de niveau recherche, publiés ou non, émanant des établissements d'enseignement et de recherche français ou étrangers, des laboratoires publics ou privés. 


\title{
Robust Trajectory Planning of Under-Actuated Cable-Driven Parallel Robot with 3 Cables
}

\author{
Edoardo Idà, Sébastien Briot, and Marco Carricato
}

\begin{abstract}
Cable-driven parallel robots (CDPRs) are under-actuated if they use a number of cables smaller than the degrees of freedom $(D o F)$ of the end-effector $(E E)$. For these robots, the constraint deficiency on the $E E$ may lead to undesirable $E E$ oscillations along the path that it is supposed to track. This paper proposes a trajectory-planning method for underactuated CDPRs which is robust against dynamic-model uncertainties or parameter variation, aiming at minimizing $E E$ oscillations along a prescribed path. Oscillation reduction and robustness are achieved by means of Zero-Vibration Multi-Mode Input Shaping and Dynamic Scaling of a reference trajectory. Simulation results show the effectiveness of the method on a 3-cable 6-DoF robot.
\end{abstract}

Key words: Cable-Driven Parallel Robot, Under-actuated Systems, Trajectory Planning, Input Shaping, Multi-Mode Systems, Dynamic Scaling

\section{Introduction}

Cable-driven parallel robots ( $C D P R s$ ) employ cables in place of rigid-body links in order to control the end-effector $(E E)$ pose. A $C D P R$ is underactuated if the number of cables is smaller than the number of the $E E$ degrees of freedom $(D o F s)$. This means that only a sub-set of the generalized coordinates of the $E E$ can be controlled, while the others are determined by the system dynamics, possibly leading to undesirable $E E$ oscillations. The use of $C D P R s$ equipped with a limited num-

Edoardo Idà · Marco Carricato

Dept. of Industrial Engineering, University of Bologna, Bologna, Italy

e-mail: \{edoardo.ida2,marco.carricato\}@unibo.it

Sébastien Briot

CNRS, Laboratoire des Sciences du Numérique de Nantes (LS2N), Nantes, France

e-mail: Sebastien.Briot@ls2n.fr 
ber of cables is justified in several applications, in which the task to be performed requires the control of a limited number of $E E D o F s$ or a limitation of mobility is acceptable to enhance workspace accessibility or decrease system cost. In recent years, increasing effort has been devoted to investigate the kinematics $[1,3]$ and dynamics $[5,6,10]$ of these manipulators.

The trajectory-planning problem for point-to-point motions of under-actuated $C D P R s$ has proven to be a major challenge, seldom studied. Idà et al. [6] presented a method to design rest-to-rest motions with prescribed path and motion time, for a generic under-actuated $C D P R$ whose dynamic model is perfectly known. Hwang et al. [5] presented a method based on Multi-Mode Zero-Vibration InputShaping (MMZVIS) to conveniently shape the input motion laws of a 3-cable $C D P R$ performing trajectories with a limited variation of the robot natural frequencies. Both theoretical and experimental results showed effectiveness of either methods, but robustness to model uncertainties was never considered.

In this paper, we propose a trajectory-planning method for point-to-point motions of an under-actuated 3-cable CDPRs that is robust against the variation of configuration-dependent parameters in the robot dynamic model, which affects the robot natural frequencies. Robustness is achieved by combining MMZVIS and Dynamic Scaling $(D S)$ of the reference trajectory. Section 2 introduces the robot model, and a methodology to compute its natural frequencies based on [5]. Section 3 describes the novel trajectory-planning method. Section 4 reports the results of numerical simulation, and Section 5 draws conclusions.

\section{CDPR Modelling}

Consider a 6-DoF CDPR composed of an $E E$ coupled to the base by 3 actuated cables. $O x y z$ is an inertial frame, $P x^{\prime} y^{\prime} z^{\prime}$ is a mobile frame attached to the $E E$ (Fig. 1a). The platform pose is described by $\mathbf{p}$, the position vector of $P$, and $\mathbf{R}$, a rotation matrix parametrized by the Euler angles $\boldsymbol{\epsilon}=[\phi, \theta, \chi]^{T}$ according to the Tait-Bryan xyz convention. The platform generalized coordinates are $\mathbf{q}=[\mathbf{p}, \boldsymbol{\epsilon}]^{T}$.

The $i$-th cable, modelled as massless and inextensible, is guided into the workspace from point $B_{i}$, described by $\mathbf{b}_{i}$ in $O x y z$, by a swivel pulley (see [6] for modelling details) and it is attached to the platform at point $A_{i}$ (Fig. 1a). $G$ is the platform center of mass. The coordinates of $G$ and $A_{i}$ are described in the mobile frame by vectors ${ }^{P} \mathbf{r}^{\prime}$ and ${ }^{P} \mathbf{a}_{i}^{\prime}$, and in the inertial frame by $\mathbf{r}=\mathbf{p}+\mathbf{r}^{\prime}=\mathbf{p}+\mathbf{R}^{P} \mathbf{r}^{\prime}$ and $\mathbf{a}_{i}=\mathbf{p}+\mathbf{a}_{i}^{\prime}=\mathbf{p}+\mathbf{R}^{P} \mathbf{a}_{i}^{\prime}$. Vector $\boldsymbol{\rho}_{i}=\mathbf{a}_{i}-\mathbf{b}_{i}$ is tangent to the pulley, and the geometrical constraint imposed by the $i$-th cable on the platform is:

$$
\boldsymbol{\rho}_{i}^{T} \boldsymbol{\rho}_{i}-\left[l_{i}-\widehat{B_{i} D_{i}}\right]^{2}=0
$$

where $l_{i}$ is the total cable length, comprising the rectilinear length $\left\|\boldsymbol{\rho}_{i}\right\|$ and the $\operatorname{arc} \widehat{B_{i} D_{i}}$ wrapped onto the pulley. The time derivatives of Eq. (1) leads, after some computation to [6]: 


$$
\mathbf{J}_{i} \dot{\mathbf{q}}=\dot{l}_{i}, \quad \dot{\mathbf{J}}_{i} \dot{\mathbf{q}}+\mathbf{J}_{i} \ddot{\mathbf{q}}=\ddot{l}_{i}
$$

where $\mathbf{J}_{i}$ is the $i$-th row of the analytic Jacobian of the system:

$$
\mathbf{J}_{i}=\left[\hat{\boldsymbol{\rho}}_{i}^{T}-\hat{\boldsymbol{\rho}}_{i}^{T} \tilde{\mathbf{a}}_{i}^{\prime} \mathbf{H}\right], \quad \mathbf{H}=\left[\begin{array}{ccc}
1 & 0 & s_{\theta} \\
0 & c_{\phi} & -s_{\phi} c_{\theta} \\
0 & s_{\phi} & c_{\phi} c_{\theta}
\end{array}\right]
$$

(.) denotes a unit vector, (.) denotes the skew-symmetric representation of the vector product.

The dynamic model of the CDPR emerges from the platform dynamics, subject to the cable constraints. $m$ is the $E E$ mass, ${ }^{P} \mathbf{I}_{G}$ its constant inertia matrix about $G$ in $P x^{\prime} y^{\prime} z^{\prime}, \mathbf{I}_{G}=\mathbf{R}^{P} \mathbf{I}_{G} \mathbf{R}^{T}$ the inertia matrix in $O x y z$, and $\mathbf{I}_{P}=\mathbf{I}_{G}-m \tilde{\mathbf{r}}^{\prime} \tilde{\mathbf{r}}^{\prime}$ its representation about $P$. If $\mathbf{f}_{G}$ and $\mathbf{m}_{G}$ are the resultant external force and moment about $G$, the platform equilibrium about $P$ leads to [6]:

$$
\begin{gathered}
\mathbf{M} \ddot{\mathbf{q}}-\mathbf{s}+\mathbf{J}^{T} \boldsymbol{\tau}=\mathbf{0}_{6}, \quad \mathbf{J}^{T}=\left[\mathbf{J}_{1}^{T} \mathbf{J}_{2}^{T} \mathbf{J}_{3}^{T}\right] \\
\mathbf{M}=\left[\begin{array}{rr}
m \mathbf{I}_{3} & -m \tilde{\mathbf{r}}^{\prime} \mathbf{H} \\
-m \mathbf{H}^{T} \tilde{\mathbf{r}}^{T} & \mathbf{H}^{T} \mathbf{I}_{P} \mathbf{H}
\end{array}\right], \quad \mathbf{s}=\left[\begin{array}{c}
m\left(\tilde{\mathbf{r}}^{\prime} \dot{\mathbf{H}}+\tilde{\boldsymbol{\omega}} \tilde{\mathbf{r}}^{\prime} \mathbf{H}\right) \dot{\boldsymbol{\epsilon}}+\mathbf{f}_{G} \\
\mathbf{H}^{T}\left[-\left(\mathbf{I}_{P} \dot{\mathbf{H}}+\tilde{\boldsymbol{\omega}} \mathbf{I}_{P} \mathbf{H}\right) \dot{\boldsymbol{\epsilon}}+\mathbf{m}_{G}+\tilde{\mathbf{r}}^{\prime} \mathbf{f}_{G}\right]
\end{array}\right]
\end{gathered}
$$

where $\boldsymbol{\tau}=\left[\tau_{1}, \tau_{2}, \tau_{3}\right]^{T}$ contains the cable tensions, and $\mathbf{I}_{3}$ and $\mathbf{0}_{6}$ are the $(3 \times 3)$ identity matrix and the $(6 \times 1)$ null vector, respectively. The generalized coordinates can be partitioned in 3 actuated coordinates $\mathbf{q}_{a}$ and 3 unactuated coordinates $\mathbf{q}_{u}$, whose time evolution is determined by the system dynamics driven by the actuated variables. According to this partition, Eq. (4) can be written as:

$$
\left[\begin{array}{ll}
\mathbf{M}_{a a} & \mathbf{M}_{a u} \\
\mathbf{M}_{u a} & \mathbf{M}_{u u}
\end{array}\right]\left[\begin{array}{c}
\ddot{\mathbf{q}}_{a} \\
\ddot{\mathbf{q}}_{u}
\end{array}\right]-\left[\begin{array}{l}
\mathbf{s}_{a} \\
\mathbf{s}_{u}
\end{array}\right]-\left[\begin{array}{l}
\mathbf{J}_{a} \\
\mathbf{J}_{u}
\end{array}\right] \boldsymbol{\tau}=\mathbf{0}_{6}
$$

Given the under-actuated nature of the system, cable tensions can be algebraically eliminated from Eq. (6), leading to the system internal-dynamics equation:

$$
\mathbf{A} \ddot{\mathbf{q}}_{a}+\mathbf{U} \ddot{\mathbf{q}}_{u}-\mathbf{s}^{\prime}=\mathbf{0}_{3}
$$

where:

$$
\mathbf{A}=\mathbf{M}_{u a}-\mathbf{J}_{u} \mathbf{J}_{a}^{-1} \mathbf{M}_{a a}, \quad \mathbf{U}=\mathbf{M}_{u u}-\mathbf{J}_{u} \mathbf{J}_{a}^{-1} \mathbf{M}_{a u}, \quad \mathbf{s}^{\prime}=\mathbf{s}_{u}-\mathbf{J}_{u} \mathbf{J}_{a}^{-1} \mathbf{s}_{a}
$$

If $\mathbf{q}_{a}$ and its time derivatives are assigned, $\mathbf{q}_{u}$ can be determined by solving Eq. (7) with an assigned initial condition. In case the initial condition is a stable equilibrium pose, it may be evaluated as in [3]. Equation (7) may also be used to determine the $E E$ oscillation natural frequencies around equilibrium configurations. To do so, $\dot{\mathbf{q}}_{a}$ and $\ddot{\mathbf{q}}_{a}$ can be isolated in Eq. (2) and the result substituted into Eq. (7), which can then be linearized about an equilibrium configuration $\mathbf{q}_{0}$ :

$$
\mathbf{M}_{u}\left(\mathbf{q}_{0}\right) \Delta \ddot{\mathbf{q}}_{u}+\mathbf{K}_{u}\left(\mathbf{q}_{0}\right) \Delta \mathbf{q}_{u}=\mathbf{0}_{3}
$$


(a)

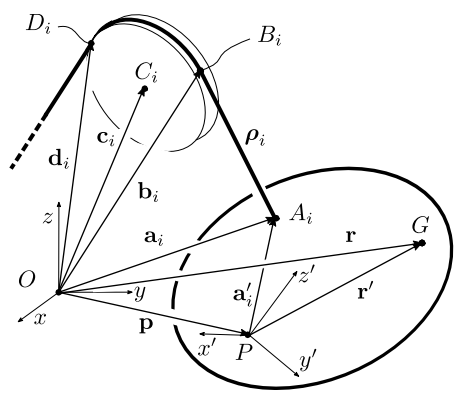

(b)

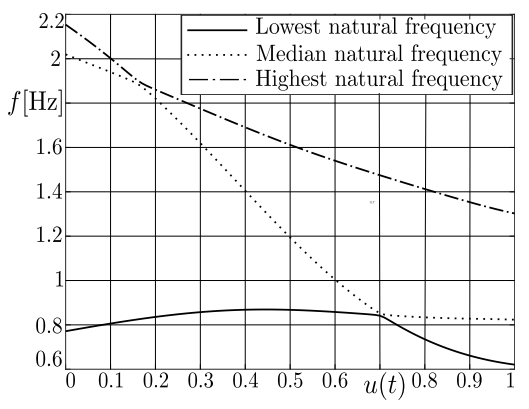

Fig. $1 C D P R$ properties: (a) Geometric model, (b) natural frequencies along a path

where $\Delta \mathbf{q}_{u}=\mathbf{q}_{u}-\mathbf{q}_{u, 0}$ is the deviation from the equilibrium of the unactuated variables, $\mathbf{M}_{u}\left(\mathbf{q}_{0}\right)$ and $\mathbf{K}_{u}\left(\mathbf{q}_{0}\right)$ are $(3 \times 3)$ symmetric positive-definite matrices (constant for an assigned $\mathbf{q}_{0}$ ) whose derivation can be found in [5]. Natural frequencies may be determined as the eigenvalues of matrix $\mathbf{M}_{u}\left(\mathbf{q}_{0}\right)^{-1} \mathbf{K}_{u}\left(\mathbf{q}_{0}\right)$, and it is clear that they change as $\mathbf{q}_{0}$ varies through the workspace (see Fig. 1b, corresponding to the path $\mathbf{q}_{0}(u)$ followed by the robot described in Section 4$)$.

\section{Trajectory Planning by Input Shaping and Dynamic Scaling}

When planning a trajectory of duration $T$ of the actuated coordinates $\mathbf{q}_{a}$, it may be convenient to separately design its geometric path, namely $\mathbf{q}_{a}=\mathbf{q}_{a}(u)$, and the motion law $u(t)$, with $u(0)=0$ and $u(T)=1$ [2]. If the path is assigned, we need to determine the motion law only. In the case of line-segment paths, which are the ones considered in this paper for the sake of simplicity, the trajectory connecting two set-points $\mathbf{q}_{a, s}$ and $\mathbf{q}_{a, f}$ is:

$$
\mathbf{q}_{a}(t)=\mathbf{q}_{a, s}+\left(\mathbf{q}_{a, f}-\mathbf{q}_{a, s}\right) u(t)
$$

A method for the design of $u(t)$ allowing the residual oscillations of $\mathbf{q}_{u}$ to be eliminated, when both the motion time $T$ and the path geometry are prescribed, was presented in [6], which suffers though from two drawbacks: the motion-law computation time is a priori unbounded (thus, it may be large), and it depends on the perfect knowledge of system parameters. Multi-Mode Zero-Vibration Input Shaping (MMZVIS) was instead used in [5] to shape actuated-coordinate trajectories; however, in order to reduce the delay time introduced by $I S$, a non-robust version of the latter was employed, leading to satisfactory results only in case of motions in a horizontal plane, where the natural frequencies of the robot at hand were only slightly variable. 
(a)

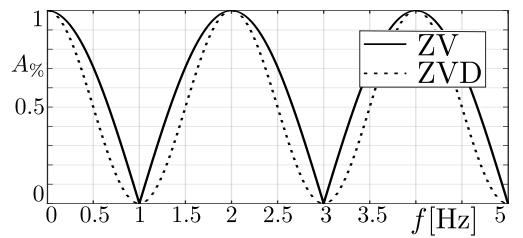

(b)

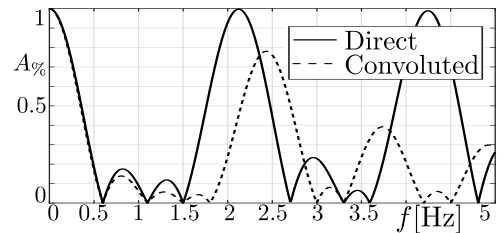

Fig. 2 (a) ZV and ZVD IS for $f=1 \mathrm{~Hz}$, (b) Direct and Convoluted IS for $f_{j}=0.6,1.1,1.5 \mathrm{~Hz}$

IS is an approach dedicated to the reduction of oscillations of second-order linear dynamic systems, and it is well-known for its simplicity [7]. Its implementation requires the convolution of a series $S(t)$ of impulses, called the input shaper, with a reference signal. By denoting the convolution operator with $*$, the input shaping of the trajectory in Eq. (10) is given by:

$$
\mathbf{q}_{a}(t) * S(t)=\mathbf{q}_{a, s}+\left(\mathbf{q}_{a, f}-\mathbf{q}_{a, s}\right)(u(t) * S(t)), \quad S(t)=\sum_{i=1}^{k} A_{i} \delta_{i}\left(t-t_{i}\right)
$$

where $\delta_{i}\left(t=t_{i}\right)=1, \delta_{i}\left(t \neq t_{i}\right)=0, A_{i}$ is the impulse amplitude, $t_{i}$ is the time at which it occurs, and $k$ is the number of impulses. The time delay added by the $I S$ is simply $t_{d}=t_{k}$, the time location of the $k$-th impulse. The pairs $\left(A_{i}, t_{i}\right)$ can be determined by setting to zero the amplitude $A_{\%}(f)$ of the Fourier trasform of $S(t)$, for an assigned frequency $f$ and $k$ impulses (Zero-Vibration or ZV IS [7], Fig. 2a):

$$
A_{\%}(f)=\sqrt{\left(\sum_{i=1}^{k} A_{i} \cos \left(2 \pi f t_{i}\right)\right)^{2}+\left(\sum_{i=1}^{k} A_{i} \sin \left(2 \pi f t_{i}\right)\right)^{2}}=0 \Longrightarrow\left(A_{i}, t_{i}\right)
$$

By definition, an input shaper is able to eliminate oscillations at the given frequency $f$ (and some multiples of it), and to reduce the amplitude of oscillation associated with every other frequency (see to the graph $\mathrm{ZV}$ in Fig. 2a). If the shaper is supposed to eliminate the oscillations at $\mu$ frequencies $f_{j}(j=1, \ldots, \mu)$, two techniques can be employed, which result in a different number of impulses and time delays [8]. The so-called direct method always uses the minimum number of impulses $k_{d i r}=1+\mu$, whereas the so-called convolved method leads to $k_{\text {conv }}=2^{\mu}$. Usually, $t_{d, d i r}<t_{d, \text { conv }}=\sum_{j=1}^{\mu} 1 /\left(2 f_{j}\right)$ but $A \%$ is slightly higher when it is not zero [8] (see Fig. $2 \mathrm{~b}$ for $f_{j}=0.6,1.1,1.5 \mathrm{~Hz}, \mu=3$ ). On the practical side, a convolved $I S$ is easy to determine, because it results from the convolution of $\mu Z V$ shapers (each one with 2 impulses), which can be computed analytically, whereas a direct $I S$ has to be numerically computed by imposing Eq. (12) to be satisfied simultaneously at $f_{1}, \ldots, f_{\mu}$, which requires $1+\mu$ impulses only (one impulse more for each additional frequency in the shaper). It should be noted that the minimum number of impulses of a direct shaper is always preferable for realtime implementation, since the amount of time required for the calculation of 
$u(t) * S(t)$, for each $t$, and thus trajectory computational complexity, increases with the number of impulses in the shaper.

The natural frequencies of a system are not always precisely known, due to either uncertainty in the dynamic-model parameters or a variation of its internal configuration (both cases occur to a robot). Loosely speaking, a trajectory is robust against natural-frequency uncertainty or variation if the amplitude spectrum of $\mathbf{q}_{a}(t) * S(t)$ is limited over some frequency range. In line-segment paths, for assigned set-points, only $u(t) * S(t)$ is responsible for the location of maximum and minimum values of this amplitude spectrum. If $U(f)$ is the amplitude of the Fourier trasform of $u(t)$, the amplitude spectrum of $u(t) * S(t)$ is $U(f) A_{\%}(f)$.

Limiting the amplitude of $U(f) A_{\%}(f)$ over a frequency range around a given frequency $f_{0}$ can be achieved by Robust IS, which uses additional impulses, and thus variables $\left(A_{i}, t_{i}\right)$, to either set $A_{\%}\left(f_{0}\right)=\partial A_{\%}\left(f_{0}\right) / \partial f=\ldots=\partial^{h} A_{\%}\left(f_{0}\right) / \partial f^{h}=0$ (ZV-Derivative ZVD IS [7], Fig. 2a), or set $A_{\%}(f)=0$ in frequencies neighboring $f_{0}$ (Extra-Insensitive EI IS [9]). In either case, the amplitude of $U(f) * A_{\%}(f)$ is flattened, and thus limited, around $f_{0}$. The addition of impulses, though, augments the time delay of the shaper, thus increasing the trajectory duration, and its computational complexity. As an alternative way to obtain similar robustness results on $U(f) A_{\%}(f)$ without the need to modify the $I S$, we propose to use Dynamic Scaling (DS) of the reference trajectory [4]. An optimal value of $T$ (as well as other trajectory parameters), for a fixed motion law profile (trapezoidal velocity, polynomial, etc..), can be determined by setting $U(f)$, or equivalently $\ddot{U}(f)=-(2 \pi f)^{2} U(f)$, to zero for the assigned frequency $f$. This strategy has the additional advantage of determining an upper bound for the total robusttrajectory duration, $T+t_{d}$. As an example, for a trapezoidal velocity profile, with $\alpha T$ acceleration and deceleration durations $(0<\alpha \leq 0.5), \alpha$ and $T$ can be determined by setting:

$$
\ddot{U}(f)=\frac{2}{(1-\alpha) \alpha(\pi T)^{2} f}\|\sin [(1-\alpha) \pi f T] \sin [\alpha \pi f T]\|=0
$$

By considering two frequencies $f_{1}$ and $f_{0}$, such that $f_{1} \geq f_{0}$, and setting the arguments of the two sine functions to $\pi$, we obtain $\alpha_{o p t}=f_{0} /\left(f_{1}+f_{0}\right)$ and $T_{\text {opt }}=\left(f_{1}+f_{0}\right) /\left(f_{0} f_{1}\right)$, where $T_{o p t}$ is strictly decreasing with $f_{1}$. It is interesting to notice that, for $f_{1} \rightarrow f_{0}, \alpha_{o p t, s}=0.5$ and $T_{o p t, s}=2 / f_{0}$, while, for $f_{1} \rightarrow+\infty$, $\alpha_{o p t, l}=0$ and $T_{o p t, l}=1 / f_{0}$. This in turn means that the optimal time is bounded by the lowest frequency and, for any $f, \alpha$ is always well defined. Moreover, total robust trajectory duration is bounded by: $T_{o p t}+t_{d} \leq T_{o p t, s}+t_{d, c o n v}$

\section{Numerical Example}

To verify the effectiveness of the combination of $I S$ and $D S$, we consider a linear trajectory of a 6-DoF 3-cable $C D P R$ with a trapezoidal velocity motion law. Model parameters are as in [6] and are not reported here due to space limitation. Natural 
(a)

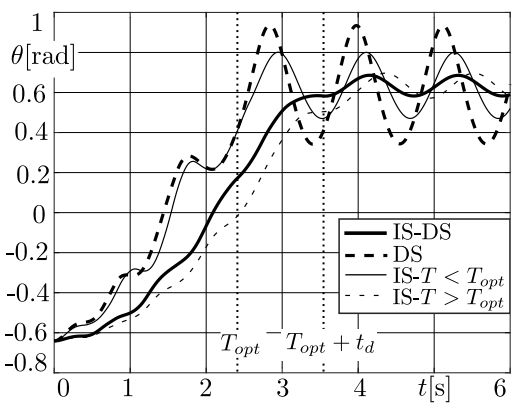

(b)

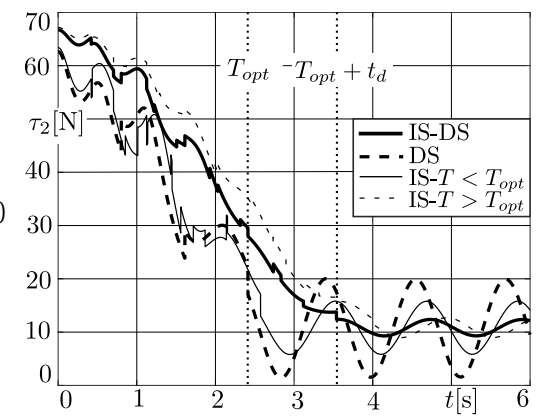

Fig. 3 Computed variables with critical oscillatory behaviors: (a) $\theta$, (b) $\tau_{2}$

frequencies along the path between $\mathbf{q}_{s}=[0.793,1.180,-0.208,0.058,-0.641,0.042]$ and $\mathbf{q}_{f}=[-0.826,1.104,-1.424,-0.041,0.634,0.002]$ (units in $\mathrm{m}$ and rad) vary as in Fig. 1b. The equilibrium value of $\tau_{2}$ in $\mathbf{q}_{f}$ is very small, and potential $E E$ oscillation in this configuration could lead to cable slackness. Since the system has 3 frequency spectra, an IS with $\mu=3$ is used, as in [5], but a direct method (with 4 impulses) is employed, and the corresponding frequencies are heuristically selected as the minimum, maximum, and median ones from Fig. 1b, i.e. $f=0.621,1.247,2.154 \mathrm{~Hz}$. In addition, since $U(f)$ is decreasing with $f$ and only 2 frequencies can be employed for the determination of $\alpha_{\text {opt }}$ and $T_{\text {opt }}$, the minimum and median ones are considered for $D S$. These choices lead to:

$$
I S: \begin{gathered}
A_{1}=A_{4}=0.2965, A_{2}=A_{3}=0.2035 \\
t_{1}=0 \mathrm{~s}, t_{1}=0.42 \mathrm{~s}, t_{3}=0.705 \mathrm{~s}, t_{4}=1.125 \mathrm{~s}
\end{gathered} \quad D S: \begin{gathered}
\alpha_{\text {opt }}=0.332 \\
T_{\text {opt }}=2.413 \mathrm{~s}
\end{gathered}
$$

The parameters in Eq. (14) are used to compute trajectory in Eq. (11), where the actuated variables are selected as the position $\mathbf{p}$ of the $E E$ reference point $\left(\mathbf{q}_{a, s}\right.$ and $\mathbf{q}_{a, f}$ are then the first 3 elements of $\mathbf{q}_{s}$ and $\mathbf{q}_{f}$, respectively). Equation (7) is numerically solved with assigned initial conditions $\mathbf{q}_{u}=[0.058,-0.641,0.042] \mathrm{rad}$ and $\dot{\mathbf{q}}_{u}=[0,0,0] \mathrm{rad} / \mathrm{s}$, in order to determine the evolution of the unactuated variables along the assigned trajectory. This step allows one to assess oscillations, but also to check for cable slackness. In case these specifications are not satisfactorily met, an intuitive solution may be to robustify $I S$ : oscillations are naturally reduced and the increase in time delay bounds cable tensions near their static equilibrium values. Most critical oscillatory variables are reported in Fig. 3 for four trajectories: the first one is planned according to $D S$ only, the second one is an IS version of the first one (IS-DS), the third and fourth ones are IS versions of trapezoidal motion laws with $\alpha=1 / 3$ (commonly employed value) and $T=0.6 T_{o p t}<T_{o p t}\left(I S-T<T_{o p t}\right)$ and $T=1.2 T_{o p t}>T_{o p t}\left(I S-T>T_{o p t}\right)$, respectively. It is clear that the use of IS leads to a more limited oscillatory behavior, and smaller oscillations occur for an increasing $T$ (since the amplitude of $U(f)$ is decreasing with $T$ ). However, on the practical side, $T$ should be as small as possible, and IS may not lead to satisfactory results if $T$ is too small. Basically, $D S$ 
helps in the choice of the best value of $T$ (and $\alpha$, for a trapezoidal velocity profile) that optimizes the transition time and limits the oscillatory behavior. Additionally, cable slackness due to oscillations is avoided with $I S$ - DS (see Fig. 3b, where $\boldsymbol{\tau}$ is computed from Eq. (6)). In the end, if critically low oscillations are required, total transition time can always be increased by means of robust $I S$.

\section{Conclusion}

In this paper, we proposed the combined use of $I S$ and $D S$ for the robust trajectory planning of under-actuated CDPRs. Simulation results were presented for a 6-DoF 3-cable $C D P R$ moving along a linear path with a trapezoidal velocity motion law. Results are satisfactory, as oscillations are appreciably reduced, when compared to a trajectory planned with $D S$ only. In the future, smoother motion laws with a larger number of parameters and generic geometric paths will be considered, in order to avoid cable tension discontinuities.

Acknowledgements The authors thank V. Mattioni for the help in preparing the illustrations.

\section{References}

1. Berti, A., Merlet, J.P., Carricato, M.: Solving the direct geometrico-static problem of underconstrained cable-driven parallel robots by interval analysis. The International Journal of Robotics Research 35(6), 723-739 (2016)

2. Biagiotti, L., Melchiorri, C.: Trajectory planning for automatic machines and robots. Springer Science \& Business Media (2008)

3. Carricato, M., Merlet, J.P.: Stability analysis of underconstrained cable-driven parallel robots. IEEE Transactions on Robotics 29(1), 288-296 (2013)

4. Hollerbach, J.M.: Dynamic Scaling of Manipulator Trajectories. Journal of Dynamic Systems, Measurement, and Control 106(1), 102-106 (1984)

5. Hwang, S.W., Bak, J.H., Yoon, J., Park, J.H.: Oscillation reduction and frequency analysis of under-constrained cable-driven parallel robot with three cables. Robotica p. 1-21 (2019)

6. Idà, E., Bruckmann, T., Carricato, M.: Rest-to-rest trajectory planning for underactuated cable-driven parallel robots. IEEE Transactions on Robotics 35(6), 1338-1351 (2019)

7. Singer, N.C., Seering, W.P.: Preshaping Command Inputs to Reduce System Vibration. Journal of Dynamic Systems, Measurement, and Control 112(1), 76-82 (1990)

8. Singhose, W., Crain, E., Seering, W.: Convolved and simultaneous two-mode input shapers. IEE Proceedings - Control Theory and Applications 144(6), 515-520 (1997)

9. Singhose, W.E., Porter, L.J., Tuttle, T.D., Singer, N.C.: Vibration Reduction Using MultiHump Input Shapers. Journal of Dynamic Systems, Measurement, and Control 119(2), 320-326 (1997)

10. Zarei, M., Aflakian, A., Kalhor, A., Masouleh, M.T.: Oscillation damping of nonlinear control systems based on the phase trajectory length concept: An experimental case study on a cable-driven parallel robot. Mechanism and Machine Theory 126, 377 - 396 (2018) 